\title{
Application of New Adaptive Higher Order Neural Networks in Data Mining
}

\author{
Shuxiang $\mathrm{Xu}$ \\ School of Computing and IS, \\ University of Tasmania, Launceston, \\ Tasmania 7250, Australia. \\ Email: Shuxiang.Xu@utas.edu.au
}

\begin{abstract}
This paper introduces an adaptive Higher Order Neural Network (HONN) model and applies it in data mining such as simulating and forecasting government taxation revenues. The proposed adaptive HONN model offers significant advantages over conventional Artificial Neural Network (ANN) models such as much reduced network size, faster training, as well as much improved simulation and forecasting errors. The generalization ability of this HONN model is explored and discussed. A new approach for determining the best number of hidden neurons is also proposed.
\end{abstract}

\section{Introduction}

Data Mining is an analytic process designed to explore data (usually large amounts of data) in search of consistent patterns and/or systematic relationships between variables, and then to validate the findings by applying the detected patterns to new subsets of data. The ultimate goal of data mining is prediction - and predictive data mining is the most common type of data mining and one that has the most direct business applications. The process of data mining usually consists of three stages: (1) the initial exploration, (2) model building or pattern identification with validation/verification, and (3) deployment. Data mining tools can answer questions that traditionally are too time-consuming to resolve. They scour databases for hidden patterns, finding predictive information that experts may miss because it lies outside their expectations. One of the most commonly used techniques in data mining, Artificial Neural Network (ANN) technology offers highly accurate predictive models that can be applied across a large number of different types of problems [1-3].

ANNs are computing tools that simulate the structure and operation of the human brain. They simulate many of the human brain's most powerful abilities such as sound and image recognition and data mining, and more importantly, the ability to generalize by observing examples (eg, forecasting based on existing situation). ANNs establish their own model of

\author{
Ling Chen \\ Information Services, \\ Department of Health and Human Services, \\ Hobart, Tasmania 7000,Australia. \\ Email: ling.chen@dhhs.tas.gov.au
}

a problem based on a training process (with a training algorithm). Conventional ANN models have been widely used in various applications, however, they suffer from several drawbacks such as large network size and slow training $[4,5]$.

To overcome these limitations some researchers have proposed the use of Higher Order Neural Networks (HONNs) [6, 7]. HONNs are networks in which the net input to a computational neuron is a weighted sum of products of its inputs. Such neuron is called a Higher-order Processing Unit (HPU) [8]. It is known that HONN's can implement invariant pattern recognition [9, 10]. [11] shows that HONN's have impressive computational, storage and learning capabilities. [12] studies the approximation and learning properties of one class of recurrent HONNs and applies these architectures to the identification of dynamical systems.

Adaptive HONNs are HONNs with adaptive activation functions. Such activation functions are adaptive because there are free parameters in the activation functions which can be adjusted (in the same way as connection weights) to adapt to different problems. In [13], an adaptive activation function is built as a piecewise approximation with suitable cubic splines that can have arbitrary shape and allows them to reduce the overall size of the neural networks, trading connection complexity with activation function complexity. In [14], real variables a (gain) and b (slope) in the generalized sigmoid activation function are adjusted during learning process. A comparison with classical ANNs to model static and dynamical systems is reported, showing that an adaptive sigmoid (ie, a sigmoid with free parameters) leads to an improved data modeling.

This paper is organized as follows. In Section 2 we propose a new adaptive HONN model with an adaptive activation function. Section 3 addresses the issue of optimizing the number of hidden layer neurons, one of the key issues yet to be resolved. In Section 4 we conduct experiments to justify our new adaptive HONN model. Section 5 gives a summary of this report as well as directions for future work. 


\section{New Adaptive HONNs}

Adaptive HONNs are HONNs with adaptive activation functions. The network structure of an adaptive HONN is the same as that of a multi-layer ANN. That is, it consists of an input layer with some input units, an output layer with some output units, and at least one hidden layer consisting of intermediate processing units (see next section on the number of hidden units). We will only use one hidden layer as it has been mathematically proved that ANNs with one hidden layer is a universal approximator [15]. Usually there is no activation function for neurons in the input layer and the output neurons are summing units (linear activation), the activation function in the hidden units is an adaptive one. Our adaptive activation function has been defined as the following:

$$
\Psi(x)=A 1 \cdot e^{-B 1 \cdot x^{2}}+A 2 \cdot \sin (B 2 \cdot x)
$$

where $A 1, B 1, A 2, B 2$ are real variables which will be adjusted (as well as weights) during training.

In our experiments (Section 4) we use an HONN learning algorithm that is based on an improved steepest descent rule [16] to adjust the free parameters in the above adaptive activation function (as well as connection weights between neurons). We will see that such approach provides more flexibility and better data mining ability for our adaptive HONN model.

\section{Number of Hidden Units for Training Adaptive HONNs}

Optimizing the number of hidden layer neurons for an ANN to solve a practical problem remains one of the unsolved tasks in this research area. Setting too few hidden units causes high training errors and high generalization errors due to under-fitting, while too many hidden units results in low training errors but still high generalization errors due to over-fitting. It is argued that the best number of hidden units depends in a complex way on: the numbers of input and output units, the number of training cases, the amount of noise in the targets, the type of hidden unit activation function, the training algorithm, etc [17]. A dynamic node creation algorithm for ANNs is proposed in [18]. [19] proposes an approach which is similar to [18] but removes nodes when small error values are reached. In [20] an algorithm is developed to optimize the number of hidden nodes by minimizing the mean-squared errors over noisy training data.

In this paper we propose an approach for determining the best number of hidden nodes based on [21], which reports that, using ANNs for function approximation, the rooted mean squared (RMS) error between the well-trained neural network and a target function $\mathrm{f}$ is shown to be bounded by

$$
O\left(\frac{C_{f}^{2}}{n}\right)+O\left(\frac{n d}{N} \log N\right)
$$

where $n$ is the number of hidden nodes, $d$ is the input dimension of the target function $f, N$ is the number of training pairs, and $C_{f}$ is the first absolute moment of the Fourier magnitude distribution of the target function $f$. The two important points of (3.1) are the approximation error and the estimation error between the well-trained neural network and the target function. For this research we are interested in the approximation error which refers to the distance between the target function and the closest neural network function of a given architecture (which represents the simulated function). To this point, [21] mathematically proves that, with $n \sim C_{f}(N /(d \log N))^{1 / 2}$ nodes, the order of the bound on the RMS error is optimized to be $O\left(C_{f}((d / N) \log N)^{1 / 2}\right)$.

Based on the above result, we can conclude that if the target function $f$ is known then the best number of hidden layer nodes (which leads to a minimum RMS error) is

$$
n=C_{f}(N /(d \log N))^{1 / 2}
$$

Note that the above equation is based on a known target function $f$.

However, in most practical cases the target function $f$ is not known, instead, we are usually given a series of training input-output pairs. In these cases, [21] suggests that the number of hidden nodes may be optimized from the observed data (training pairs) by the use of a complexity regularization or minimum description length criterion [22]. This is a criterion which reflects the trade-off between residual error and model complexity and determines the most probable model (in this research, the HONN with the best number of hidden nodes). Based on this, when $f$ is unknown we use a complexity regularization approach to determine the constant $C$ in the following

$$
n=C(N /(d \log N))^{1 / 2}
$$

The approach is to try an increasing sequence of $C$ to obtain different number of hidden nodes, train an ANN for each number of hidden nodes, and then observe the $n$ which generates the smallest RMS error (and note the value of the $C$ ). The maximum of $n$ has been proved to be $N / d$ [22]. Please note the difference between the equation (3.2) and the equation (3.3): in (3.2), $C_{f}$ depends on a known target function $f$, which is usually unknown (so (3.2) is only a theoretical approach), whereas in our approach as shown in (3.3), $C$ is a constant which does not depend on any function.

Based on our experiments conducted so far we have 
found that for a small or medium-sized dataset (with less than 5000 training pairs), when $N / d$ is less than or close to 30 , the optimal $n$ most frequently occurs on its maximum, however, when $N / d$ is greater than 30 , the optimal $n$ is close to the value of $(N /(d \log N))^{1 / 2}$.

\section{Experiments}

Our first experiment is to use our adaptive HONN to simulate and then forecast the Total Taxation Revenues of Australia. Figure 4.1 shows the financial data downloaded from the Australian Taxation Office (ATO) web site. For this experiment monthly data between Sep 1969 and June 1999 are used (358 data points). Based on our new approach, the optimal number of hidden layer neurons for this experiment is $n=5$. Additionally, the average training time is only 3.5 seconds.

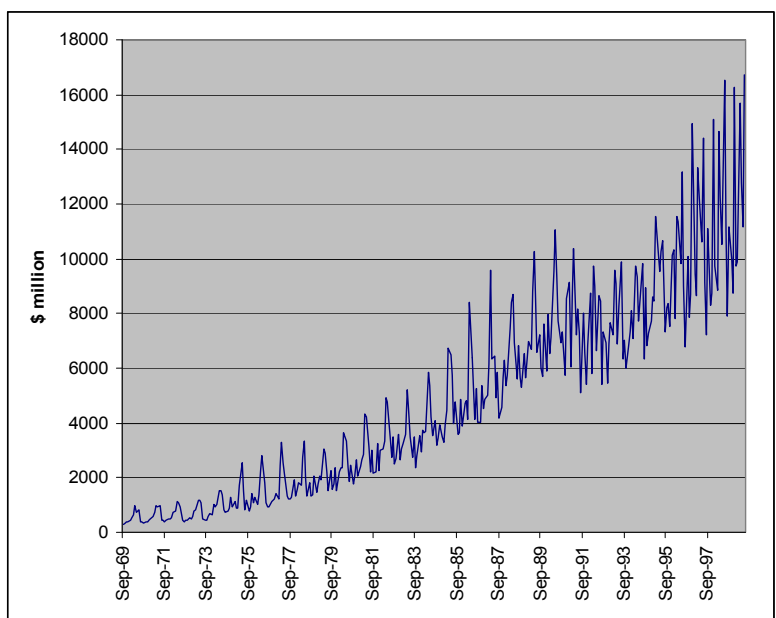

Figure 4.1. Total Taxation Revenues of Australia (\$ million) (Sep 1969 to June 1999)

After the adaptive HONN (with 5 hidden layer units) has been well trained over the training data pairs, it is used to forecast the taxation revenues for each month of the period July 1999 - June 2000. Then the forecasted revenues are compared with the real revenues for the period, and the overall RMS error reaches $4.12 \%$. To verify that for this example the optimal number of hidden layer neuron is 5 , we try to apply the same procedure by setting the numbers of hidden layer neurons to $4,6,8$, and 12 , which results in overall RMS errors of $6.76 \%, 7.19 \%, 9.88 \%$, and $10.33 \%$, respectively. Some cross-validation method is used for this experiment: the training data set is divided into a training set made of $70 \%$ of the original set and a validation set made of $30 \%$ of the original set. To verify the advantages of our adaptive HONN model we establish a conventional ANN with the sigmoid activation function (and one hidden layer) for the same experiment. With the same number of hidden neurons and the same training set (and the same crossvalidation), the conventional ANN reaches an overall RMS error of $14.98 \%$, with an average training time of 11.9 seconds. Further experiments show that to get to the same RMS error of $4.12 \%, 14$ hidden nodes have to be set for a conventional ANN.

For our second experiment, a dataset containing information of different cars built in the US, Europe, and Japan is trained using our Adaptive HONN to determine car fuel economy (MPG - Miles Per Gallon) for each vehicle. There are a total of 392 samples in this data set with 9 input variables and 1 output. The dataset is from UCI Machine Learning Repository (2007) [23]. The output is the fuel economy in MPG, and the input variables are:

- number of cylinders

- displacement

- horsepower

- weight

- acceleration

- model year

- $\quad$ Made in US? $(0,1)$

- $\quad$ Made in Europe? $(0,1)$

- $\quad$ Made in Japan? $(0,1)$

The dataset is divided into a set containing 353 samples for training, and a set containing 39 samples for forecasting (or generalization). A cross-validation mechanism is adopted which splits the training set into 2 sections to train our Adaptive HONN (75\% for training and $25 \%$ for validation). Based on our approach, the optimal number of hidden layer neurons for this experiment is $n=4$. Again it's easy to verify whether this is the optimal number simply by setting a different number of hidden layer neurons and then compare the simulation and forecasting errors. After our adaptive HONN (with 4 hidden layer units) has been trained over the training data samples, it is used to generalize over the 39 forecasting samples, which results in an overall RMS error of only $3.85 \%$. The average training time is only 2.9 seconds. Meanwhile, with the same number of hidden neurons and the same training set (and the same cross-validation), a conventional ANN model with the sigmoid activation function (and one hidden layer) results in an overall RMS error of $12.08 \%$, with an average training time of 9.3 seconds. Further experiments show that to get to the same RMS error of $3.85 \%, 16$ hidden nodes have to be set for a conventional ANN.

\section{Summary and Discussions}


In this paper an adaptive HONN model is introduced and applied in data mining such as forecasting government taxation revenues. Such model offers significant advantages over conventional ANNs such as much reduced network size, faster training, as well as much improved simulation and forecasting errors. A new approach for determining the best number of hidden nodes has been proposed. Compared with conventional approaches on applying ANN models in data mining, although there are more free parameters in our adaptive HONN model, training speed is increased due to a significant decrease of network size. For future work, it would be a good idea to extend the research to involve large applications which contain training datasets of over 5000 input-out pairs. Further comparison studies between our adaptive HONN and other ANN approaches should also be conducted to demonstrate the advantages of our approach.

\section{References}

[1] Adriaans, P., Zantinge, D., 1996, Data Mining, AddisonWesley.

[2] Han. J., Kamber, M., 2001, Data Mining: Concepts and Techniques, Morgan Kaufmann Publishers.

[3] Sarker, R. A., Abbass, H. A., Newton, C. S., 2002, Data Mining: A Heuristic Approach, Idea Group Pub./Information Science Publishing.

[4] Berry, M.J.A., and Linoff, G. 1997, Data Mining Techniques, NY: John Wiley \& Sons.

[5] Han. J., Kamber, M., 2001, Data Mining: Concepts and Techniques, Morgan Kaufmann Publishers.

[6] Redding, N., Kowalczyk A. and Downs, T., (1993). "Constructive high-order network algorithm that is polynomial time", Neural Networks, Vol.6, pp.997-1010.

[7] Zhang, M., Xu, S., Fulcher, J., (2002). Neuron-Adaptive Higher Order Neural-Network Models for Automated Financial Data Modeling, IEEE Transactions on Neural Networks, Vol 13, No. 1.

[8] Lippman, R.P. (1989) Pattern classification using neural networks, IEEE Commun. Mag., 27, 47-64.

[9] Psaltis, D., Park, C.H., Hong, J. (1988) Higher order associative memories and their optical implementations, Neural Networks, 1, 149-163.
[10] Reid, M.B., Spirkovska, L., Ochoa, E. (1989). Simultaneous position, scale, rotation invariant pattern classification using third-order neural networks, Int. J. Neural Networks, 1, 154-159.

[11] Giles, C.L., Maxwell, T. (1987) Learning, invariance, and generalization in higher order neural networks, Applied Optics, 26(23), 4972-4978.

[12] Kosmatopoulos, E.B., Polycarpou, M.M., Christodoulou, M.A., Ioannou, P.A. (1995). High-order neural network structures for identification of dynamical systems, IEEE Transactions on Neural Networks, 6(2), 422431.

[13] Campolucci, P., Capparelli, F., Guarnieri, S., Piazza, F., \& Uncini, A. (1996). Neural networks with adaptive spline activation function. Proceedings of IEEE MELECON 96, Bari, Italy, 1442-1445.

[14] Chen, C.T., Chang, W.D. (1996). A feedforward neural network with function shape autotuning, Neural Networks, 9(4), 627-641.

[15] Leshno, M., Lin, V.Y., Pinkus, A., Schocken, S. (1993). Multilayer feedforward networks with a nonpolynomial activation function can approximate any function, Neural Networks, 6, 861-867.

[16] Xu, S. (2008) Chapter XIV: Adaptive Higher Order Neural Network Models and Their Applications in Business, Artificial Higher Order Neural Networks for Economics and Business, Zhang M (ed), pp 314-329, IGI Global.

[17] Haykin, S. (1999), Neural networks: a comprehensive foundation, N.J. : Prentice Hall.

[18] Ash T., 1989, Dynamic node creation in backpropagation networks, Connection Science, Volume 1, Issue 4, pp $365-375$.

[19] Hirose Y. Yamashita I.C., Hijiya S., 1991, Backpropagation algorithm which varies the number of hidden units, Neural Networks, Vo1.4. 1991.

[20] Rivals, I., Personnaz, L., 2000, A statistical procedure for determining the optimal number of hidden neurons of a neural model, Second International Symposium on Neural Computation (NC'2000), Berlin, May 23-26 2000.

[21] Barron, A. R., (1994), Approximation and Estimation Bounds for Artificial Neural Networks, Machine Learning, (14): 115-133, 1994.

[22] Barron, A. R., Cover, T. M. (1991). Minimum complexity density estimation. IEEE Transactions on Information Theory, 37, 1034-1054.

[23] UCI Machine Learning Repository ftp://ftp.ics.uci.edu/pub/machine-learning-databases/autompg/auto-mpg.data. Accessed on 1 May 2008. 